\title{
Preface: Catalytic Aspects of Complete Oxidation of Volatile Organic Compounds
}

\author{
Stuart H. Taylor
}

Published online: 18 February 2009

(C) Springer Science+Business Media, LLC 2009

Volatile Organic Compounds (VOCs) are a wide ranging class of compounds that are diverse in their properties and chemistry, and they are not limited to any one functionality. The definition of a VOC is somewhat ambiguous, as it alters according to the source. For instance, the US EPA defines a VOC based on relative photochemical reactivity in the atmosphere, whilst under European law it is defined in terms of volatility and not reactivity. Although the definition of a VOC is unclear, there is little dispute that their release to the atmosphere can have serious deleterious affects. In the 1980s it was established that the presence of chlorinated-VOCs in the atmosphere were responsible for the thinning of stratospheric ozone, which led to the formation of the seasonal hole in the ozone layer. In addition to ozone depletion, VOCs have also been implicated in the formation of photochemical smog, increased concentration of ground level ozone, often they are potent greenhouse gasses and also toxic/carcinogenic substances.

The sources of VOC emission are diverse, and originate from both natural and anthropogenic sources. The release of naturally derived VOCs, from sources such as forests, swamps, insects and animals, are difficult to control, whilst those of anthropogenic origin can be more easily controlled. A range of BOC abatement technologies have been developed and these include thermal oxidation, catalytic oxidation, adsorption, absorption and condensation. Catalytic oxidation offers a number of advantages over competing methods as it is able to destroy the VOCs, it is less fuel intensive compared to thermal oxidation and it is versatile with the capability to treat waste streams with a wide variation of VOC concentrations and effluent flow rates. A wide range of catalysts have been investigated for complete oxidation of VOCs, and commercial catalysts based on supported precious metals and metal oxides have been developed. The diversity of VOCs and the extensive variety of their emission sources means that there still remains plenty of scope to develop better catalysts. For instance, the identification of more efficient catalysts to reduce operating costs and improve efficiency of energy utilisation remains an important research aim. Improved catalysts are also required for the oxidation of VOCs that contain heteroatoms, such as, sulphur, nitrogen and halogens.

For the manufacture and processing of chemicals there is a driving force to develop zero emission processes, but for many transformations this remains a target that has not yet been achieved. Furthermore, other sources of VOC emission, such as, combustion processes will be used for the foreseeable future. Hence, ultimately the applications for VOC oxidation catalysts may diminish in the long-term, it appears that they will still be required for applications in the medium-term. 